\title{
Doing interdisciplinarity: motivation and collaboration in research for sustainable agriculture in the UK
}

\author{
Frances Harris ${ }^{1}$, Fergus Lyon ${ }^{2}$, Sarah Clarke, \\ A later version of this paper was published as Harris, F, Lyon, F and Clarke, S (2009) Doing \\ interdisciplinarity: motivation and collaboration in research for sustainable agriculture \\ in the UK. Area 41, 4, 374 - 384 \\ ${ }^{1}$ School of Earth Science and Geography, University of Kingston.
${ }^{2}$ Centre for Enterprise and Economic Development Research, Middlesex University
${ }^{3}$ Elm Farm Research Centre.
}

Key words: Interdisciplinarity, case studies, rural, researchers, collaboration, trust

\begin{abstract}
This paper studies knowledge production in complex, collaborative research projects which brought together academics of different disciplines, research users and agricultural businesses. It takes a comparative approach, studying the interactions within interdisciplinary research teams from 10 case studies, considering the process of collaboration from initial idea through to publication. The research developed a typology of participants in these projects, and identified the motivations and challenges of each. Our results analyse the process of research teams coming together and the relationships which are built up during the research. A particular challenge identified was the building of cooperation and trust. This issue is explored alongside issues of communication, methodology, data analysis and the process of drawing and publicising conclusions.
\end{abstract}

\section{Introduction}

Interdisciplinary research has been defined as an integration of ideas from different disciplines, drawing on each others theories, research methods, and ways of viewing world (Sillitoe, 2004). The process of carrying out interdisciplinary research has been studied through many reflective pieces drawing on personal experiences. However, there have been few studies that have taken comparative approaches to examine the different motivations and challenges being faced by researchers of different disciplines and also different professional approaches. This study draws on details of 10 case study research projects each of which includes academic researchers of different disciplines, those working in commercial agricultural-based businesses (including farmers) and scientists working in third sector/not for profit organisations and agri-environmental pressure groups. Through developing a typology of different types of researchers, we are able to examine differing motivations for interdisciplinarity and the range of challenges and factors affecting how collaboration is established and maintained. 
Research on agro-ecosystems often integrates expertise from different academic disciplines, as well as utilizing the expertise of research users such as businesses, land managers, farmers and conservationists. This is both interdisciplinary as well as being transdisciplinary. This paper examines the process by which different types of researchers can navigate these diverse approaches and find ways of collaborating.

The paper answers the following research questions: What are the motivations of different types of researchers? What are the challenges these different types of researchers face in the process of initiating and carrying out interdisciplinary research? How are collaborative relationships between different types of scientists built and maintained during a project and in the longer term?

The research focuses on the motivations and challenges within interdisciplinary research and the driving force that brings such research teams together. This is followed by an analysis of the challenges faced by research groups as they negotiate their way through problem identification and goal setting, research proposal preparation, methodologies, data analysis and interpretation, dissemination of results, and finally consider what happens at project completion. Thus we consider the challenges along the research journey, and draws on people's experiences and hindsight to learn lessons for future projects. Particular insights concern the role of donors in stimulating interdisciplinary research, the role of collaboration and trust in establishing an effective research team and how the demands of the Research Assessment Exercise ${ }^{1}$ and academic career structures conflict with interdisciplinary research.

This paper adds to current research in five ways. Firstly, the research studies a selection of complex, collaborative research projects on agro-ecosystems in the UK which brought together different types of researchers (academics, commercial scientists, not for profit organisation scientists and innovative businesses such as farmers). Thus it considers research which spans the boundaries of academic disciplines, as well as the boundaries of academia and environmental management practitioners (farmers, land managers, environmental NGOS and conservation groups).

Secondly, it takes a comparative approach, studying the interactions within interdisciplinary research teams from 10 case studies, to gather empirical data concerning the process from initial idea through to publication and dissemination.

Thirdly, a typology of type of researcher participants in the case study projects is also identified. The results demonstrate the range of motivations and challenges, identifying differences based on both discipline and type of researcher.

Fourthly, through our research, we also pay particular attention to the way that trust is developed among collaborating research group teams. Our results analyse the process

\footnotetext{
${ }^{1}$ The Research Assessment Exercise (RAE) is the UK's system of assessing and rating university research departments. Success depends largely on publications and research income. This RAE is carried out approximately every 5 years, resulting in the production of league tables of university departments. The results of the RAE have a huge influence over the level of core funding departments receive in the future.
} 
of research teams coming together and the relationships which are built up during the research. Issues of trust are central to understanding the interdisciplinary process.

Finally, the research approach used is novel too. An interdisciplinary research team planned and developed this research. When interviewing collaborators in the case study interdisciplinary research projects, four members of each research team were interviewed. The interviews were always conducted by a pair of researchers from this project's research team - ensuring that there was always one natural and one social scientist from this research project collecting information from the case study projects. In this way, an interdisciplinary approach was embedded within the methodology of our own research project.

\section{Motivations and challenges of interdisciplinary research}

The concept and practice of interdisciplinary research has become increasingly common over the last decade. This paper aims to examine the motivations of different types of researchers in these interdisciplinary contexts. It is argued that the increase in interdisciplinary research is a response to an appreciation of the complexity of research questions, especially those concerning environmental and sustainability issues. "Environmental research has unique characteristics because it encompasses both social and ecological dimensions" (Scott et al., 1999, pg 4). Furthermore, problems such as sustainability, people-environment relations, technology innovation and risk assessment require interdisciplinary approaches (Thompson-Klein, 2004). Sustainability and environmental concerns are both seen as issues where traditional disciplinary approaches are unable to provide sufficient breadth to address and research critical issues (Russell et al., 2007; Balsiger, 2004; Scott et al., 1994), and hence these are some of the driving forces behind the move towards more interdisciplinary research. There are also calls for more participatory approaches to engage practitioners: businesses, communities, NGOs and end-users of research.

Research that crosses disciplinary boundaries can be referred to as multi-, inter-, or trans-disciplinary. These terms are not always used in the same way in the literature (see, among others, Wickson et al., 2006, Thompson-Klein, 2004, Fry, 2001, Tress and Tress, 2001; Sillitoe, 2004, Lawrence and Depres, 2004, Ramadier, 2004, Pohl, 2008) '. In this paper, we use the following definitions:

- Multidisciplinary research tackles issues from several disciplines. There is no attempt to integrate the research results.

- Interdisciplinary research is carried out at the boundaries of existing disciplinary knowledge, and in the interstices between disciplines. The aim is to develop new knowledge and new approaches to research and thinking based on the integration and further development of ideas from individual disciplines.

- Trans-disciplinary research goes beyond academic disciplines to include other, non-academic groups, such as farmers, other businesses, government and policy makers, and the public. These seek to span both disciplinary and professional boundaries. Such an integrated approach requires crossing the boundaries between different knowledges (disciplines) and between scientific knowledge and tacit knowledge (Lawrence and Depres, 2004). 
This papers' second aim is to examine the challenges faced by different types of researchers attempting interdisciplinary research. A criticism leveled at some past experiences of research across disciplines has been the lack of engagement between people of different disciplines with each person following different perspectives and priorities.

As researchers have embraced interdisciplinary research, this has provided the scope of writing about the nature of interdisciplinary work and the challenges of achieving this (Sillitoe, 2004; Tress, Tress and Fry, 2005; Thompson-Klein, 1990 and 2004; Fry, 2001). Much of this work has focussed on the differences between disciplines, and the barriers which interdisciplinary research teams face as they try to come together. Some of this has involved work written by the interdisciplinary researchers themselves, reflecting on personal research journeys through the development of interdisciplinary research partnerships (Bracken and Oughton, 2006 Jones and Macdonald, 2007, Fry, 2001).

Overcoming these disciplinary boundaries can be a challenge prior interdisciplinary research, particularly in the early stages of projects. Crow (in Sillitoe, 2004) states that the three key factors in establishing an interdisciplinary research team are parity, reciprocity, and a common language. Yet our research shows that establishing such a balanced rapport can be a process fraught with difficulties.

Focusing on an issue-based rather than discipline based approach to research makes it easier to bring together an interdisciplinary research team. The agreement on the overall aim of the research project, and how to take this desire for joint research forwards, requires negotiation and agreement on research priorities. However, the underlying premises each discipline brings to research are different, and there can be conflict at this early stage. These relationships have to be seen in the context of the current academic environment where individuals controlling research resources can influence the career opportunities of others, especially contract or non tenured researchers. Tress, Tress and Fry (2005) identify some of the tensions related to meeting both the applied project outputs and scientific expectations.

The combination of language, culture and research methods define individuals as being from one discipline or another. Differences in the way disciplines frame the objects of their study (ontological heritage) can jeopardize cooperation and yet problem definition is the key to developing a common vision of the project (Brewer, 1999) and the intellectual agenda to develop new techniques and communities of research practice.

The language used informs researchers' views of the world, and understanding of research problems (Sillitoe, 2004). Bracken and Oughton (2006) argue that common understanding derived from shared languages plays a vital role in enhancing the relations of trust that are necessary for effective interdisciplinary working. Indeed, Sillitoe (2004) points out the merits of being able to argue one's academic case comfortably with those of other disciplines, being familiar with their terminology and vocabulary. Interdisciplinary work has been considered analogous to cultural journeys, with the need to consider oneself as a "visitor to other disciplines" 
(Galmiche-Tejeda, 2004), "landing on alien shores, you must begin to acquire the local culture, not with the aim of denying your origins, but so that you can gain the full respect of the natives" (Simon, 1992). Implicit in this is the understanding that the development of mutual language and understanding takes time (Bracken and Oughton 2006).

Alongside these issues is a concern over the perceived quality of interdisciplinary research. Interdisciplinarity requires methodological flexibility (Balsiger, 2004), and many researchers are afraid that it will result in compromise, leaving research which fails to satisfy the quality criteria of any one discipline (Lau and Pasquini, 2004). On the other hand, it has been argued that "border work" (Horlick-Jones and Sime, 2004) is not only where research can engage in practical "real life" problems, but is also the environment where truly novel and innovative ideas and research practices are generated (Sillitoe, 2004, Ramadier, 2004).

These challenges demonstrate the need for interdisciplinary researchers to build relationships with others from different viewpoints and approaches. The nature of collaborative relationships is explored in the third aim of this paper. While collaboration has been identified as a key issue in previous studies (eg Fry, 2001; Tress and Tress, 2001; Cummings and Kiesler, 2005), this paper develops our understanding of the processes underpinning collaboration drawing on a range of literature related to trust within and between organisations. We argue that interdisciplinarity has to be seen as a relationship and underpinning such relationships are issues of trust and power (Lyon, 2006). There is a need to understand how trust is used and built; not just assuming it appears when there are incentives (Granovetter, 1985; Mollering, 2006). We define trust here as the expectation that people will act as expected and people will make themselves vulnerable to opportunism from others. A dictionary definition of trust is "a firm belief in the reliability, truth or strength of a person; a confident expectation; and a reliance on the truth of a statement without examination" (Oxford English Dictionary). Trust operates when there is confidence in other agents, despite uncertainty, risk and the possibility for them to act opportunistically (Misztal, 1996: 18; Gambetta, 1988:218).

However, relationships may also be shaped by power relations. The most common form of power exerted over others is through sanctions such as legal contracts. However, Klein Woolthuis et. al (2005) found that trust often precedes contracts and the two reinforce and complement each other. Power can also be exerted less explicitly through influencing the views and perceptions of others (Lukes, 1974). Latour (1987) examines the processes of exerting power with regard to scientist and non-scientist interaction, demonstrating how more powerful parties can enrol others into their views.

The process of building trust has received relatively little attention in the academic literature with only the work of Newell and Swan (2000) examining trust in university research networks. Inter-organisation relationships can also be shaped by trust in a third party or intermediary (Lyon, 2000). Individuals who can bridge different professional cultures play key roles as boundary spanners (Williams, 2002) although this requires a range of competences including diplomatic skills. 


\section{Methodology}

An interdisciplinary research team which included a human geographer, an environmental scientist, a biologist, an agronomist and a rural sociologist worked together to develop the research. An initial sampling frame of 50 existing and completed research projects was identified. From this, 10 cases were selected to ensure that the case studies included a range of different approaches to interaction, different degrees of collaboration and different disciplines (see table 1). Criteria for selection included:

Involvement of researchers from different institutions

The research team came together for the specific research project ranging from three months to five years.

The projects involved both interdisciplinary and transdisciplinary interactions The selection represents a cross section of types of partnership, subjects and types of research. Details of the case studies are shown in table 1.

Within each case study, face to face semi-structured interviews were carried out with a minimum of four individuals having differing roles, including farmers, advisors, researchers and funders. These were selected purposefully to ensure a cross section of people with different views. There is potential bias from this relatively small sample although the in-depth qualitative nature of the approach did not allow for a larger sample. The topic guide for the interviews was used to collect background information on the individual being interviewed and their organisation. Detailed probing was used to explore how they are going about their research and learning, their interaction with other stakeholders, and external factors that have shaped this interaction.

Particular attention was given to exploring what happened at 'critical incidents' such as meetings to discuss the research objectives or results (Chell, 1998). This approach allowed detailed information to be collected on the 10 case studies and explore the processes of building relationships, while recognising the constraints of using single interviews for collecting data on processes occurring over long periods of time. Observations of people's reactions to questions were also important in terms of documenting information that might be tacit knowledge or gut reactions to particular issues (Ambrosini and Bowman, 2001).

To ensure consistency in data collection each interview involved one of the authors. In order to gain greater insights into the different professional and disciplinary cultures each interview was conducted by a pair of academics from contrasting disciplines (e.g. natural and a social scientist). An important part of the research process involved cross disciplinary meetings and training to build capacity in interviewing and to gain an understanding of different disciplinary approaches and norms.

Analysis was carried out through the comparison of differences between case studies of the same type and between different types of groups (Yin, 2003). This involved careful analysis of interview responses as well as noting the reactions of respondents such as the use of laughter and non-verbal responses to particular questions. While there is potential bias from the small sample and the role of the interviewers/data 
analysers, validity and accuracy was promoted by using a range of techniques (interviewing, observations, informal discussions), combined with a sampling of cases allowing cross case comparison and the cross checking of issues from multiple sources ('triangulation').

\section{The processes of doing interdisciplinary research in the 10 case studies}

This section presents the findings of this research and discusses the implications of our results with reference to previous studies.

\section{Motivations to undertake interdisciplinary and transdisciplinary research}

Table 1 presents a summary of the 10 case studies on which this research is based. They represent a range of types of projects, with differing partnerships, geographical location and funders.

\section{Insert Table 1 here Table 1 Brief description of case studies}

In six of the ten cases studies, interviewees claimed that it was the funders (predominantly DEFRA, either alone or as part of a LINK ${ }^{2}$ programme) who had demanded an interdisciplinary or transdisciplinary approach to the research. This contrasted with anecdotal comment in the literature denying the demand for interdisciplinary research from research councils (e.g. Lau and Pasquini, 2004). In one instance the demand for interdisciplinary research was made clear through specific requirements stated in an invitation to tender from DEFRA. In other instances, interdisciplinarity was a requirement of research councils. Of the remaining 4 projects, funding agencies made it clear that the nature of the research question demanded an interdisciplinary research team.

None of the teams had worked together before, although some individual members may have collaborated together in previous research projects. While most of the research teams had come together themselves, in one case, the research team was formed by the funder selecting which aspects of research were desired, and then asking those researchers to form an research team and work together towards set goals. This implies a greater degree of dialogue between funders and researchers as recommended by Tress, Tress and Fry (2005) who found that funders in the past seldom giving practical advice.

Insert table 2 here

Table 2 Roles of funders in influencing the motivations of researchers to work across disciplinary boundaries in each of the case studies

Table 2 shows how the varied motivations researchers are strongly shaped by funders. The research projects brought together researchers, businesses, and

\footnotetext{
${ }^{2}$ The Department for Food, Environment and Rural Affairs (DEFRA) funds LINK research projects to encourage researchers to interact with the agricultural industry. A criterion of LINK is that the funding provided by Defra is matched by in-kind or cash contributions from businesses to ensure that outcomes are both scientifically rigorous and industry relevant.
} 
practitioners from differing backgrounds. In the initial stages of the research projects, each of these had their own goals with respect to the project, which had to be taken into consideration in the project design, and which shaped the nature and degree of interdisciplinarity. Within teams there were differing views and resistance from some to interdisciplinary working. There was the potential for the development of both alliances and conflicts between differing viewpoints.

The results from all the case studies lead to the development of a typology of researchers each of which having differing motivations in their research:

Commercial technology company scientists want to have ideas that can be converted into profitable businesses for customers.

> Pressure group scientists want to disseminate research rapidly via membership newsletters or popular press.

Academic researchers may want results to be more rigorously statistically tested so that they can be disseminated through publication in peer-refereed journals - a process which takes many years.

Contract researchers have specific funding pressures and tight deadlines in which to complete particular projects.

To these types of researchers a final category can be added of farmers who through their practice and involvement in the research were part of the research process to different degrees in each of the projects studied. Each type of actor brings particular characteristics, which are reflected in both their motivations to be involved in interdisciplinary research, and in the particular challenges that interdisciplinary research poses. The specific challenges for each type of researcher varied. The case studies show that there is potential for conflict if differing goals and aspirations are not managed carefully.

\section{Challenges of interdisciplinary and transdisciplinary research}

Agreeing on project aims:

The combination of funders, academics, farmers, NGOs, contract researchers and businesses lead to high emotions. These were summarized aptly by several of those interviewed:

"Projects have been hijacked for academic purposes and they are not very practical... from the point of view of what academics want out of a project, they want papers, they want to build empires, they want post-docs and therefore if a project does go down an academic route the problem is that it can lead to disenfranchisement of the commercial partners, they become less interested and they put less in. I won't join quasi-academic LINK projects with little or no commercial work." (Commercial technology company scientist) 
"This project on хххххx was difficult because of the difference in perspectives. There were attempts to push them together but it was difficult at times as some of the senior people had different priorities and it was difficult in the steering group because they couldn't, how can I say, there was not too much flexibility, it was very much towards what they needed to do to justify where the money ... came from. The more multi- or inter-disciplinary they saw, the less scientific robustness they felt it was." (Academic researcher)

"It is always a compromise in terms of value for money in scientific terms. And some of these projects is science by consensus. The LINK programme has multiple funding and so therefore has multiple objectives. " (Contract researcher)

Career progression. The structures of research institutions and career pathways within academia are a limiting factor for interdisciplinary research. Among academics, there was the added difficulty of finding mutually respected journals for publication by multi-disciplinary teams, especially given the demands of peers, departments, and career progression. Even where teams were working well, members faced pressures from those not involved in the research, who viewed the interdisciplinary endeavour differently. Academic researchers faced pressure from others in their department (particularly in the context of finalising RAE submissions), and also in their peer community. A particular challenge is that other academics working within their own disciplines may not appreciate the methdolocial challenges and advances made by their interdisciplinary peers (Fry, 2001). There was a concern that stepping beyond the boundaries of their discipline to engage with other disciplines would result in compromising professional integrity (through adapting methodologies and approaches) and subsequent loss of intellectual rigour. This could result in a loss of credibility within the discipline, affecting their reputation, and therefore with implications for future funding. This is particularly important for those who are at the earlier stages of their career such as contract researchers and lecturers. As a former head of department (now retired) put it "Middle managers (like Deans) are driven by performance yardsticks, and so instruct their 30-40 year old staff members to aim for a 4* academic profile, defined by publications, grants, and workshops / events to increase their profile. Interdisciplinarity does not score... " (Academic researcher)

Interviewees reported that it is difficult to build a career on interdisciplinarity, and this has been further emphasized by the RAE, which encourages a focus on the core of a discipline (research questions, and publication in research journals) and sees venturing out into collaborative work with other disciplines as compromise and of less academic value. This still occurs despite the growth, in some areas, of high ranking interdisciplinary journals (Fry, 2001).

More established academics interviewed reported that it was easier for them to engage in interdisciplinary research than those in early stages of their careers. This was found to be particularly true for contract research staff, who have yet to secure permanent posts within University departments, and so are more likely to want to stay within an identifiable core of their discipline. Furthermore, without job security, these researchers are likely to move on to the best job offer, and without on-going research funds, are unable to stay within the interdisciplinary research team which has been developed through earlier projects. 
Publication of results:

Another challenge concerned publishing with two distinct issues becoming apparent in the analysis. Firstly there is the desire of non-academics in transdisciplinary projects to publicise the results immediately, which is contrasted with the more measured academic approach of repetition of research to ensure accuracy, submission to journals, and peer review. Secondly, there is frequently debate among academic researchers about the type and quality of journal in which to publish results.

In two cases there was conflict as researchers in an NGO wanted to use the trade and popular press for rapid dissemination of results, whereas academics wanted greater replication, statistical rigour and to publish in peer-reviewed journals. This seemed an unnecessarily slow process to NGOs who were keen to show their members that they were using funds for their benefit, and able to make timely recommendations and influence policy.

When the possibility arises of research results turning into a marketable, profitable product, there is a new urgency with respect to the results, and possibly an element of secrecy too, which creates a new opportunity for challenge and conflict among the members of the research team. An added consideration in publicising results was whether everyone benefited from the publicity. In one case study there was pressure to hold back on publicising the results when the products of one company involved were found to be performing poorly:

" $х x х x x$ did restrict project results because it made them look bad in the first year but they allowed them to become available in the third year when subsequent results showed it wasn't that bad" (Contract researcher)

There was concern over the perceived quality of interdisciplinary research journals. These were seen by many as inferior publication channels when compared to "core" or "gold standard" journals within disciplines, and this has implications in the RAEdominated culture within UK academia. A distinction needs to be made between interdisciplinary journals covering similar disciplines and those with a broader remit. Some interdisciplinary journals that do not straddle such wide ranges of disciplines have established themselves.

\section{Integration of methodology}

Once the overall research agenda was set in the case study research projects, deeper debates followed concerning methodology. Research projects which linked economic, social and natural sciences faced a major challenge agreeing how to go about research. "They usually work to very strict protocols so it's difficult to be flexible." (Farm manager). Underpinning these discussions were questions of relative rigor of different disciplinary approaches, and whether a hierarchy of disciplines existed.

"I think the expression of design by committee applied to some extent here. Different people had different ideas so there was an element of negotiation of what was in there, so compromises had to be made."(Farm manager) 
Discussions were further exacerbated by fears that a poor research approach resulting from compromise would produce results which were of no practicable use (whether for publication, commercial development, or policy-oriented).

Conflict was found between disciplines when researchers from one discipline perceived a tacit "hierarchy" of methodological approaches. These distinctions were found between natural science vs. social science methodologies as well as between quantitative and qualitative approaches. This underpinned discussions of methods to be used, data collected, the relative weight of results, and what conclusions could be drawn from the study.

In the process of analysing data, there were also reports of debate over how results would be interpreted. Researchers coming from different perspectives and concentrating on different factors can have very different interpretations of similar aspects. In two cases the interpretation of the data about the impact of a particular conservation measure was found to differ with one partner organisation stating it was good for the environment and another that it was bad. This is partly due to the emphasis on different interpretations of the environment and the preoccupation with specific aspects of the environment (such as a particular species) by some researchers.

One compromise approach to integration of methodologies is to allow disciplines to carry out their own rigorous research, and hope to integrate data at a later stage. However this compromise results in subsequent problems. When researchers have done independent studies (in a multi- rather than inter-disciplinary approach), someone has to bring the work together to interpret the studies as a whole.

"The biggest challenge is to have a true partnership and trying to get things intertwined rather than having separate bits. I am trying to get people to write together at the moment for this report. I usually get lots of individual reports from the different organisations but the reader has to make the connections. I don't think we're getting the most out of the information. I am trying to get everyone together. Get them to edit it, not me to interpret their work. I get stacks of individual reports and I don't think we capture everything in there. It takes longer, editorial meetings, making decisions together, listening to each other's work." (Funder of research) A big issue is whether the research team works together to draw out the conclusions, or whether an individual is faced with the task. In one case the funders forced the different researchers to work together: "We are making them produce joint reports, so it is quite a facilitation exercise; it is not the way they are used to working."(Funder of research)

\section{Team building and trust}

In each case study, the research team was composed of people from companies, farms, NGOs and research institutions, and thus at the outset were a disparate group, in terms of backgrounds, language or jargon, and common working practices. This presented particular challenges, particularly where team members had only been involved in mono-disciplinary academic research with others of the same background (language, methodology, research norms). When research calls demanded that new 
interdisciplinary teams were formed, there was little time to develop modes of cooperation and trust prior to embarking on proposal development. In one extreme case, funders had reviewed several proposals and then drawn together what they saw as the best strands of each and asked those involved to form a new project team. More often, the demands of funding encouraged people to draw on their own contacts to form an interdisciplinary research team. In three of the cases, a trusted colleague vouched for new people who were brought into the group. The best teams had worked together in the past, knew each other beforehand and already established trust. As one researcher commented "You have to work with people that you know will deliver" (technology company scientist).

Respondents reported that trust is required as there is reliance on others to carry out the research according to expectations (although these may vary between professional cultures based on different norms of doing the research). Furthermore trust is required to ensure that individuals follow different norms regarding the use of results (in press releases, publications or using intellectual property in future commercial activities). The varied norms and different values between disciplinary cultures resulted in tensions particularly with respect to the quality of work, publishing results before some team members felt it was suitable and "hogging the limelight". Trust was reported to be particularly important when working in less well known areas and crossing disciplines where research partners may be less comfortable and feel more at risk from external criticism.

\section{Conclusions}

Interdisciplinarity is central to answering geographical questions such as occur in agro-ecosystems and land use research that cuts across traditional disciplinary boundaries and also meets the needs of non-research stakeholders who do not follow the disciplinary boundaries of academia. Among the academic community it is widely believed that funding for interdisciplinary research is rare (e.g. Tress et al, 2005, Lau and Pasquini, 2004). However, our findings show that, for many projects, the demands of those providing research funding was one of the driving forces which encouraged an interdisciplinary approach. Furthermore, while accepted as the driving force, research funders were also criticised as only having one-off programmes and short-term projects which did not allow research teams to continue their interdisciplinary research. The government departments commissioning research were also criticised for using their power to be overly prescriptive. This contrasts to the findings of previous research that found that funders to be unclear of what they wanted (Tress and Tress, 2001) and demonstrates the need to look at the different approaches of each type of funding.

The work identified a typology of scientists involved in interdisciplinary research projects. These included technical company scientists, contract researchers, pressure group scientists and university academics. Each had slightly different priorities, agendas and standards depending on the different motives for carrying out research. These motives included developing profitable business opportunities, lobbying policy makers or enhancing personal careers. 
Many spoke of the challenges of bringing interdisciplinary teams together. These included differences in disciplinary perspectives, language and the challenge of understanding different disciplinary perspectives of other team members. Setting up research priorities and methodologies was difficult, with several of those interviewed referring to 'design by committee' and projects being 'hijacked' by particular members of the team, either senior scientists, or academic researchers, to meet their particular needs. There is a need to consider the power relations within teams and the roles of project leaders who can pursue their own agendas through designing research and writing final reports. A big challenge was learning to value the different types of information each discipline might produce, such as natural scientists valuing social science information, and the concerns of those who work with quantitative data when faced with qualitative data. Agreeing on the final conclusions of projects was also difficult, with people referring to 'science by consensus'.

Towards the end of projects, researchers have had to overcome difficult decisions concerning where and how to publish results. Some want peer-reviewed articles in journals, others more widely-distributed, easily understood outputs for farmers, businesses or the wider public. Agreement on the level of robustness of the information, as well as the venue for publication, caused concern in some projects. Institutional pressures are a large factor in interdisciplinary and transdisciplinary research. Interviewees reported that it is difficult to build a career on interdisciplinarity, and this has been further emphasized by the RAE, which encourages a focus on the core of a discipline (research questions, and publication in research journals) and sees venturing out into collaborative work with other disciplines as compromise and of less academic value. More established academics interviewed reported that it was easier for them to engage in interdisciplinary research than those in early stages of this career.

Another outcome of this research was a clear indication of how important it is to develop a team that can work together. In each of the cases, issues of co-operation were based on the development of interpersonal trust between organisations. The issue of physical distance between research collaborators was identified in previous studies (Cumings and Kiesler, 2005, Bracken and Oughton, 2006) but was not referred to by participants in this research. Trust is also shown to be based on having potential sanctions over them, either through contractual controls, but, most importantly for the teams examined, in terms of peer pressure. In three cases, key people played the role of intermediary or boundary spanner, bringing together researchers or institutions which might not have worked together without the presence of someone trusted by all parties i.e. a guarantor. Other teams could build on relationships coming out of existing research communities. In bridging disciplinary boundaries the cases demonstrated the need to recognize the different norms of research in different disciplines and professions.

Alongside other researchers, this work found that the establishment of a good interdisciplinary research team requires time to develop relationships, build-up trust, and identify good working practices. As research teams evolve and build up trust, they become stronger, through better communication (development of common language), are able to experiment with integration of new research approaches, and are more likely to be open in debate concerning the integration, interpretation and implication 
of research results. If teams are left to disband due to a lack of further funding, this emergent body of knowledge, skills and trust is often lost.

Acknowledgements: This research was funded by the Rural Economy and Land Use programme. The authors wish to thank Prof. Paul Sillitoe for valuable comments on the manuscript.

\section{References}

Ambrosini V, and Bowman C, 2001 Tacit knowledge: Some suggestions for operationalization Journal of Management Studies 38 (6) 811-829

Balsiger PW, 2004 Supradisciplinary research practices: history, objectives and rationale. Futures, 36, (4), 407-421

Bracken L J and Oughton EA 2006. "What do you mean?” The importance of language in developing interdisciplinary research, Transactions of the Institute of British Geographers, 31 (3). 371-382

Brewer GD, 1999 The challenges of interdisciplinarity. Policy Sciences 32, 327-339

Chell E, 1998 Critical Incident Technique, in Symon G and Cassell C eds Qualitative Methods and Analysis in Organisational Research-a Practical Guide, Chapter 4, 51-72, London: Sage

Cummings JN and Kiesler S 2005 Collaborative research across disciplinary and organisational boundaries. Social Studies of Science 35 (5)703-722.

Fry, G, 2001 Multifunctional landscapes - towards transdisciplinary research. Landscape and Urban Planning 57 , 159-168

Galmiche-Tejeda A, 2004 Who is interdisciplinary? Two views, two goals, professionals and farmers. Interdisciplinary science reviews 29 (1) 77-95

Gambetta, D. 1988 Can we trust trust? in Gambetta, D ed Trust: Making and Breaking Cooperative Relations, Oxford: Blackwell, 213-37

Granovetter M, 1985 Economic action and social structure: the problem of embeddedness, American Journal of Sociology 91 (3) 481-510

Horlick-Jones T and Sime J, 2004 Living on the border: knowledge, risk and transdisciplinarity. Futures 36 (4) 441-456

Jones P and Macdonald N, 2007 Getting it wrong first time: building an interdisciplinary research relationship Area, 39 (4) 490-498

Klein Woolthuis, Hillebrand B and Nooteboom B, 2005 Trust, contract and relationship development. Organization Studies 26 (6) 813-840

Latour B, 1987 Science in action: how to follow scientist and engineers through society Cambridge M.A. Harvard University Press

Lau L and Pasquini MW, 2004 Meeting grounds: perceiving and defining interdisciplinarity across the arts, social sciences and sciences Interdisciplinary science reviews 29 (1) 49-64

Lawrence JR and Depres C, 2004 Introduction. Futures of transdisciplinarity. Futures 36 397-405

Lukes S, 1974 Power: A Radical Review London: Macmillan.

Lyon F, 2006 Managing co-operation - trust and power in Ghanaian associations Organization Studies 27 (1) 31-52

Lyon F, 2000 Trust, networks and norms: The creation of social capital in agricultural economies in Ghana World Development 28 (4) 663-682

Misztal BA, 1996 Trust Cambridge: Polity Press. 
Mollering G, 2006 Understanding trust from the perspective of sociological neoinstitutionalism, in Bachmann, R and Zaheer, A eds Handbook of Trust Research Cheltenham, Edward Elgar

Newell S and Swan J, 2000 Trust and inter-organizational networking, Human Relations 53 (10) 1287-1328

Pohl C 2008 From science to policy through transdisciplinary research Environmental Science \& Policy Volume 11, Issue 1, Pages 46-53

Ramadier R 2004. Transdisciplinarity and its challenges: the case of urban studies Futures, 36 (4) 423-439

Russell AW Wicksen F and Carew AL, 2007 Transdisciplinarity: Context, contradictions and capacity. Futures In press.

Scott A Skea J Robinson J and Shove E, 1999 Designing 'interactive' environmental research for wider social relevance. Brighton, Economic and Social Research Council Global Environmental Change Programme: 16. Special Briefing No. 4.

Sillitoe P, 2004 Interdisciplinary experiences: working with indigenous knowledge in development. Interdisciplinary science reviews 29 (1) 6-23.

Simon H, 1992 Living in interdisciplinary space. in Szendberg M ed Eminent economists: their life philosophies Cambridge: Cambridge University Press

Thompson-Klein J, 2004 Prospects for transdisciplinarity Futures 36 515-526

Thompson-Klein J, 1990 Interdiscplinarity History, theory and Practice Detroit: Wayne

Tress, B and Tress, G, 2001 Capitalising on multiplicity: a transdisciplinary systems approach to landscape research Landscape and Urban Planning 57, 3-4, 143157

Tress, B, Tress, G and Fry, G, 2005 Integrative studies on rural landscapes: policy expectations and research practice Landscape and Urban Planning 70, 15, 177-191

Wickson F Carew AL and Russell AW 2006 Transdisciplinary research: characteristics, quandaries and quality Futures 1046-1059

Williams P, 2002 The competent boundary spanner Public Administration 80 (1) 103124

Yin R, 2003 Case study research design and methods London: Sage.

Zucker L G, 1986 Production of trust: Institutional sources of economic structure, 18401920 Research in Organisational Behaviour 8, 53-111

\section{List of Tables}

Table 1 Types of disciplines and professions involved in the case studies

Table 2 Roles of funders in influencing the motivations of researchers to work across disciplinary boundaries in each of the case studies 